ISSN (e)-2347-176x ISSN (p) 2455-0450

crossrefDOI: https://dx.doi.org/10.18535/jmscr/v7i1.107

Journal Of Medical Science And Clinical Research

IGM Publication

An Official Publication of IGM Publication

Original Article

\title{
Comparative evaluation of 25-Hydroxy vitamin D levels among women with polycystic ovarian syndrome and normal women of reproductive age group attending a tertiary care hospital
}

\author{
Authors \\ Dr Rukhsana Sami ${ }^{1}$, Dr Sheikh Mohd Saleem², Dr Qazi Haroon ${ }^{3}$ \\ ${ }^{1}$ Assistant Professor, Department of Gynecology \& Obstetrics, Lal Ded Hospital \\ (Associated Hospital of Government Medical College, Srinagar \\ ${ }^{2}$ Demonstrator, Department of Community Medicine, Government Medical College, Srinagar \\ ${ }^{3}$ State Nodal Officer, Family Planning/State Immunization Officer (SEPIO), J\&K. \\ Directorate of Family Welfare, MCH \& Immunization, J\&K, India
}

\begin{abstract}
Background: Polycystic Ovary Syndrome (PCOS) has been termed as the most common endocrine entity encountered by the women of the reproductive age group. The aim of the study was to find the comparison between Vitamin D levels among Normal women and Women with PCOS in the reproductive age group at one of the largest maternity hospitals of Kashmir Valley.

Methods: The study was a cross-sectional study which was carried out at one of the largest maternity tertiary care hospitals of Kashmir Valley. Women in the reproductive age group, women diagnosed with PCOS using clinical or Ultrasound criteria. For comparison, women who attended the outpatient department for any cause other than PCOS were included in the study.

Results: The mean age of the patients was $28 \pm 8$ years in PCOS women and $27 \pm 6$ years among normal women without PCOS. Majority 99 (66\%) of the patients without PCOS were having serum fasting insulin levels within normal range while $123(82 \%)$ among PCOS were having increased levels of serum fasting insulin levels. Moreover, 148 (99\%) of PCOS patients fall under any one of the categories of Vitamin D deficiency while the reverse was true for women without PCOS.

Conclusion: Fasting serum Insulin levels and Serum 25(OH) Vitamin-D levels were significantly lower in $P C O S$ women than the normal women in the reproductive age group.

Keywords: PCOS, Ovarian Disease, 25(OH) D Levels, Insulin Levels.
\end{abstract}

\section{Introduction}

Polycystic Ovary Syndrome (PCOS) has been termed as the most common endocrine entity encountered by the women of the reproductive age group. Characteristics features of PCOS include dysfunction of ovaries which leads to oligomenorrhea and eventually anovulation, hirsutism due to hypersecreations of androgens and an ultrasound diagnosis of multiple cysts morphology of ovaries ${ }^{(1)}$. Long term risk factors of PCOS include type 2 diabetes mellitus, carcinoma endometrium and cardiovascular diseases $^{(2)}$. Vitamin D is an important precursor and plays an important role in our body especially 
in the bones. The active form of Vitamin D (25Hydroxy vitamin D) has a role in bone metabolism, calcium-phosphorus homeostasis and many other cell proliferation and differentiation processes $^{(3)}$. Vitamin D is has also an important role in reproductive system including follicular development in the ovaries by signaling antiMullerian hormone pathways ${ }^{(4)}$. PCOS, a complex disorder causes defect in the metabolism and reproductive system $^{(5,6)}$ and many studies have linked its pathogenesis to Vitamin D deficiency which eventually leads to metabolic syndrome ${ }^{(7,8)}$. Furthermore, some studies have even documented association of obesity with the decreased levels of Vitamin D in patients with $\operatorname{PCOS}^{(7,8)}$.

The deficiency of Vitamin D has been observed to be quiet common among the population and studies have found vitamin D levels as low as 20 $\mathrm{ng} / \mathrm{ml}$ in $10-60 \%$ of the adults ${ }^{(9)}$. The causes of Vitamin D deficiency include decreased skin synthesis of Vitamin D, hereditary disorders of vitamin D synthesis, kidney diseases etc. Other factors which reduced absorption from the skin include use of skin creams, skin pigmentation and skin grafts. Moreover, obesity causes reduced bioavailability of Vitamin $\mathrm{D}^{(10)}$. Many studies have compared Vitamin D Levels among normal women and women with PCOS. Some studies found no change in the levels of Vitamin $D^{(1)}$ while some showed higher or lower levels of Vitamin $\mathrm{D}^{(11,12)}$. We conducted this study with the aim to find the comparison between Vitamin D levels among Normal women and Women with PCOS in the reproductive age group at one of the largest maternity hospitals of Kashmir Valley.

\section{Material \& Methods}

Study Design \& Setting: The study was a crosssectional study which was carried out at one of the largest maternity tertiary care hospitals of Kashmir Valley.

Study Period \& Study Unit: The study was conducted for a period of 2 months from October 2018-November 2018 among normal women and women with PCOS attending outpatient department of a tertiary care hospital.

Sample Size: Arbitrarily we included 150normal women and 150 women with PCOS

Inclusion Criteria: Women in the reproductive age group, women diagnosed with PCOS using clinical or Ultrasound criteria. For comparison, women who attended the outpatient department for any cause other than PCOS were included in the study.

Exclusion Criteria: Women with known endocrine disorders, liver disease, renal disease, drug history of Vitamin D agonists or antagonists, women on Hormonal replacement therapy.

Questionnaire: A questionnaire was developed before the study to capture socio-demographic (13) and clinical details.

Procedure: The patients were grouped into normal women without PCOS and women with PCOS and were explained the objectives of the study after taking informed consent. Fasting serum insulin levels and 25 Hydroxy Vitamin D levels were measured using enzyme linked Immunosorbant Assay (ELISA) with a normal range of $75-100 \mathrm{nmol} / \mathrm{ml}$. The obtained levels of $25(\mathrm{OH}) \mathrm{D}$ were classified as $<25 \mathrm{nmol} / \mathrm{ml}$ as Severe Deficiency, 25- $49.9 \mathrm{nmol} / \mathrm{ml}$ as Deficiency, $50-74.9 \mathrm{nmol} / \mathrm{ml}$ as Insufficiency and above $75 \mathrm{nmol} / \mathrm{ml}$ as normal. The responses were entered in the Excel Spreadsheet and later analyzed using SPSS v 20.0 statistical software. Statistical Analysis: Data was presented as measures of central tendency. Categorical variables were analysed using Chi-square test. Ethical Issues: The study didn't have any ethical issues related to human or animal experiments. The confidentiality of the participants was maintained as per study protocol.

\section{Results}

The study involved a total of 300 patients grouped into women with PCOS and normal women without PCOS. The mean age of the patients was $28 \pm 8$ years in PCOS women and $27 \pm 6$ years among normal women without PCOS. Majority of 
the patients in PCOS group were overweight (28\%) and obese (51\%). Such was not the case with women without PCOS .Majority of the study participants in our study belonged to upper middle and lower middle socioeconomic class as per Kuppuswamy Socioeconomic scale update 2018 (13).[Table 1]

Table 1: Socio-demographic characteristics of the study participants

\begin{tabular}{|l|c|c|}
\hline $\begin{array}{l}\text { Age } \\
\text { Involved }\end{array}$ & $\begin{array}{c}\text { Women with } \\
\text { PCOS } \\
\mathbf{n = ( 1 5 0 )}\end{array}$ & $\begin{array}{c}\text { Normal Women } \\
\mathbf{n =}(\mathbf{1 5 0 )}\end{array}$ \\
\hline Age in Years & $54(36 \%)$ & $46(31 \%)$ \\
\hline $14-18$ & $54(36 \%)$ & $64(43 \%)$ \\
\hline $19-30$ & $32(21 \%)$ & $30(20 \%)$ \\
\hline $31-40$ & $10(7)$ & $10(7)$ \\
\hline$>40$ & & $20(13 \%)$ \\
\hline BMI & $12(8 \%)$ & $36(24 \%)$ \\
\hline Lean & $20(13 \%)$ & $46(31 \%)$ \\
\hline Normal & $42(28 \%)$ & $48(32 \%)$ \\
\hline Overweight & $76(51 \%)$ & $9(6 \%)$ \\
\hline Obese & $11(7 \%)$ & $55(37 \%)$ \\
\hline Socioeconomic status & $58(39 \%)$ & $48(32 \%)$ \\
\hline Upper (I) & $45(30 \%)$ & $28(19 \%)$ \\
\hline Upper Middle (II) & $22(15 \%)$ & $10(7 \%)$ \\
\hline Lower Middle (III) & $14(9 \%)$ & \\
\hline Upper Lower(IV) &
\end{tabular}

Fasting serum Insulin levels are mentioned in Table 2 along with serum $25(\mathrm{OH})$ D levels. Majority $99(66 \%)$ of the patients without PCOS were having serum fasting insulin levels within normal range while 123 (82\%) among PCOS were having increased levels of serum fasting insulin levels. The association was statistically significant. Moreover, 148 (99\%) of PCOS patients fall under any one of the categories of Vitamin D deficiency while the reverse was true for women without PCOS and the association was statistically significant.

Table 2: Serum Fasting Insulin Levels \& Serum $25(\mathrm{OH})$ D levels among PCOS and Non PCOS women

\begin{tabular}{|c|c|c|c|}
\hline & PCOS Cases & Normal Cases & P-Value \\
\hline \multicolumn{4}{|c|}{ Fasting Serum Insulin Levels } \\
\hline $3-9 \mathrm{miu} / \mathrm{lt}$ & $27(18 \%)$ & $99(66 \%)$ & \multirow[t]{2}{*}{$<0.001 *$} \\
\hline$>10 \mathrm{miu} / \mathrm{lt}$ & $123(82 \%)$ & $51(34 \%)$ & \\
\hline \multicolumn{4}{|c|}{ Serum $25(\mathrm{OH})$ D levels } \\
\hline $\begin{array}{l}\text { Severely } \\
\text { Deficient }\end{array}$ & $88(59 \%)$ & $20(13 \%)$ & \multirow[t]{4}{*}{$<0.001 *$} \\
\hline Deficient & $45(30 \%)$ & $40(27 \%)$ & \\
\hline In sufficient & $15(10 \%)$ & $59(39 \%)$ & \\
\hline Normal & $2(1 \%)$ & $31(21 \%)$ & \\
\hline
\end{tabular}

\section{Discussion}

The cross sectional study included 300 patients who were diagnosed PCOS cases and normal women (150 each) and inferences were drawn between the two groups. The mean age of the patients in PCOS group was $28 \pm 8$ years while mean age among non PCOS group was $27 \pm 6$ years. Most of the patients in PCOS group were found to be overweight (28\%) and obese (51\%). Such was not the case with non PCOS women group $^{(14)}$. Majority of the study participants in our study belonged to upper middle and lower middle socioeconomic class as per Kuppuswamy Socioeconomic scale update $2018^{(13)}$. In this study we compared the serum fasting Insulin levels among the two group and found that $99(66 \%)$ of the patients without PCOS were having serum fasting insulin levels within normal range while $123(82 \%)$ among PCOS were having increased levels of serum fasting insulin levels. The association was statistically significant. This is in accordance with the studies published earlier where they found that the serum fasting insulin levels among the women with diagnosed PCOS was below normal values ${ }^{(15-17)}$.

Moreover we also compared serum 25(OH) D levels among the two groups. The results indicated that the level of $25(\mathrm{OH}) \mathrm{D}$ levels were significantly lower in PCOS women than in the non PCOS women. The association between the two was statistically significant. The results of our study are with agreement with the studies published by Elida et al. ${ }^{(18)}$. In their case control study, they found that there were higher levels of $25(\mathrm{OH})$ D levels among the control group containing non PCOS women than the cases group containing PCOS diagnosed women. Another study conducted by Li et al. ${ }^{(1)}$ done on 25 women with PCOS found an inverse relationship between 25(OH) D levels and BMI. Majority (72\%) women among the cases were having Vitamin D deficiency and almost half of them with severe deficiency. This is also in accordance with the results of our study. Wehr et al ${ }^{(19)}$ also reported lower serum Vitamin D levels among women with 
PCOS compared to normal women without PCOS and their association was statistically significant. The result published by Wehr et $\mathrm{al}^{(19)}$ were in accordance with our study.

\section{Conclusion}

Fasting serum Insulin levels and Serum 25(OH) Vitamin-D levels were significantly lower in PCOS women than the normal women in the reproductive age group.

\section{Acknowledgement}

Authors would like to extend their greetings to, Head of Department, Department of Gynecology \& Obstetrics, Lal Ded Associated Hospital of Government Medical College, Srinagar for her support and continuous inspiration. Authors would like to acknowledge help of Dr. Sheikh Mohd Saleem, Demonstrator, Department of Community Medicine, Government Medical College, Srinagar for the final review of this manuscript.

Conflict of Interest None declared

Source of Funding None

\section{References}

1. $\mathrm{Li} \mathrm{H}$, Brereton $\mathrm{R}$, Anderson $\mathrm{R}$ et al. (2011): Vitamin D deficiency is common and associated with metabolic risk factors in patients with polycystic ovary syndrome. Metabolism,60:1475-1481.

2. Haoula Z, Salman M and Atiomo W (2012): Evaluating the association between endometrial cancer and polycystic ovary syndrome. Hum. Reprod., 27:1327-1331.

3. Studzinski G, McLane J and Uskokovic M (1993):Signaling pathways for vitamin D-induced differentiation: implications for therapy of proliferative and neoplastic diseases. Crit. Rev. Eukaryot. Gene Expr.,3:279-312.

4. Irani M and Merhi Z (2014): Role of vitamin $\mathrm{D}$ in ovarian physiology and its implication in reproduction: a systematic review. Fertil. Steril., 102: 460-469.
5. B. N. Kalro, T. L. Loucks, and S. L. Berga, Neuromodulation in polycystic ovary syndrome, Infertility and Reproductive Medicine Clinics of North America, 14, no. 4,529-555, (2003).

6. L. Speroff and MA. Fritz, Chronic Anovulation and The Polycystic Ovary Syndrome,in Clinical Gynecologic, Endocrinology and Infertility, 495-533, 2011, 8th ed.

7. E. Wehr, S. Pilz, N. Schweighofer, A. Giuliani, D. Kopera, T. R. Pieber, and B.Obermayer-Pietsch, Association of hypovitaminosis $\mathrm{D}$ with metabolic disturbancesin polycystic ovary syndrome, European Journal of Endocrinology, 161, no. 4, 575-582,(2009).

8. E. Wehr, O. Trummer, A. Giuliani, H.-J. Gruber, T. R. Pieber, and B. ObermayerPietsch,Vitamin D-associated polymorphisms are related to insulin resistance and vitaminD deficiency in polycystic ovary syndrome, European Journal of Endocrinology, 164,no. 5, 741749, (2011).

9. M. F. Holick, Medical progress: Vitamin D deficiency, New England Journal ofMedicine, 357, no. 3, 266-281, (2007).

10. Lips P (2010): Worldwide status of vitamin D nutrition. J .Steroid .Biochem Mol. Biol.,121:297-300.

11. Holick M (2007): Medical progress: vitamin D deficiency. New England Journal of Medicine,3: 266-281.

12. Franks S (1995): polycystic ovary syndrome. pathogenesis of PCOS. N. Engl. J. Med., 333:853-861.

13. Sheikh Mohd Saleem. Modified Kappuswamy scale updated for year 2018. Indian J Res. 2018;7(3):6-7.

14. Ngo D, Chan W, Rajendran $\mathrm{S}$ et al. Determinants of insulin responsiveness in young women: Impact of polycystic ovarian syndrome, nitric oxide and 
vitamin D. Nitric Oxide,2011. 25: 326330.

15. Mahmoudi T, Gourabi H, Ashrafi $M$ et al.Calciotropic hormones, insulin resistance and the polycystic ovary syndrome. Fertility and Sterility, 2010.93: 1208-1214.

16. Moini A, Shirzad N, Ahmadzadeh M et al.Comparison of 25-hydroxyvitamin $\mathrm{D}$ and calcium levels between polycystic ovarian syndrome and normal women. Int. J .Fertil. Steril.2015, 9: 1-8.

17. Figurová J, Dravecká I, Javorský M et al.Prevalence of vitamin D deficiency in Slovak women with polycystic ovary syndrome and its relation to metabolic and reproductive abnormalities. Wien. Klin. Wochenschr.2016,10: 1-8.

18. Elida S, Binarwan $\mathrm{H}$, Muhammad $\mathrm{F}$ et al. Vitamin D levels in women with polycystic ovary syndrome. ASPIRE Conference Proceedings, The 6thCongress of the Asia Pacific Initiative on Reproduction. KNE Medicine, 2016.125:88-95

19. Wehr E, Pilz S, Schweighofer $N$ et al.Association of hypovitaminosis D with metabolic disturbances in polycystic ovary syndrome Eur. J Endocrinol.2009 ,161:575-82. 